\title{
High-resolution chromosome painting with repetitive and single-copy oligonucleotides in Arachis species identifies structural rearrangements and genome differentiation
}

Pei Du ${ }^{1,2+}$, Lina $\mathrm{Li}^{1+}{ }^{1}$, Hua Liu', Liuyang Fu ${ }^{1,4}$, Li Qin ${ }^{1}$, Zhongxin Zhang ${ }^{1}$, Caihong Cui ${ }^{1}$, Ziqi Sun ${ }^{1}$, Suoyi Han ${ }^{1}$, Jing X X' ${ }^{1}$, Xiaodong Dai ${ }^{1}$, Bingyan Huang ${ }^{1}$, Wenzhao Dong ${ }^{1}$, Fengshou Tang ${ }^{1}$, Lifang Zhuang ${ }^{2}$, Yonghua $\operatorname{Han}^{3}$, Zengjun $\mathrm{Qi}^{2^{*}}$ and Xinyou Zhang ${ }^{1 *}$

\begin{abstract}
Background: Arachis contains 80 species that carry many beneficial genes that can be utilized in the genetic improvement of peanut (Arachis hypogaea L. $2 n=4 x=40$, genome AABB). Chromosome engineering is a powerful technique by which these genes can be transferred and utilized in cultivated peanut. However, their small chromosomes and insufficient cytological markers have made chromosome identification and studies relating to genome evolution quite difficult. The development of efficient cytological markers or probes is very necessary for both chromosome engineering and genome discrimination in cultivated peanut.

Results: A simple and efficient oligonucleotide multiplex probe to distinguish genomes, chromosomes, and chromosomal aberrations of peanut was developed based on eight single-stranded oligonucleotides (SSONs) derived from repetitive sequences. High-resolution karyotypes of 16 Arachis species, two interspecific $F_{1}$ hybrids, and one radiation-induced $\mathrm{M}_{1}$ plant were then developed by fluorescence in situ hybridization (FISH) using oligonucleotide multiplex, $45 \mathrm{~S}$ and $5 \mathrm{~S}$ rDNAs, and genomic in situ hybridization (GISH) using total genomic DNA of $A$. duranensis $(2 n=2 x=20, A A)$ and A. ipaënsis $(2 n=2 x=20, B B)$ as probes. Genomes, chromosomes, and aberrations were clearly identifiable in the established karyotypes. All eight cultivars had similar karyotypes, whereas the eight wild species exhibited various chromosomal variations. In addition, a chromosome-specific SSON library was developed based on the single-copy sequence of chromosome $6 \mathrm{~A}$ of A. duranensis. In combination with repetitive SSONs and rDNA FISH, the single-copy SSON library was applied to identify the corresponding A3 chromosome in the A. duranensis karyotype.

\footnotetext{
* Correspondence: zjqi@njau.edu.cn; haasz@126.com

${ }^{\dagger}$ Pei Du and Lina Li contributed equally to this work.

${ }^{2}$ State Key Laboratory of Crop Genetics and Germplasm Enhancement, Nanjing Agricultural University, Nanjing 210095, China

${ }^{1}$ Industrial Crops Research Institute, Henan Academy of Agricultural Sciences/

Key Laboratory of Oil Crops in Huang-Huai-Hai Plains, Ministry of Agriculture/

Henan Provincial Key Laboratory for Oil Crops Improvement, Zhengzhou

450002, Henan, China

Full list of author information is available at the end of the article
}

(c) The Author(s). 2018 Open Access This article is distributed under the terms of the Creative Commons Attribution 4.0 International License (http://creativecommons.org/licenses/by/4.0/), which permits unrestricted use, distribution, and reproduction in any medium, provided you give appropriate credit to the original author(s) and the source, provide a link to the Creative Commons license, and indicate if changes were made. The Creative Commons Public Domain Dedication waiver (http://creativecommons.org/publicdomain/zero/1.0/) applies to the data made available in this article, unless otherwise stated. 


\begin{abstract}
(Continued from previous page)
Conclusions: The development of repetitive and single-copy SSON probes for FISH and GISH provides useful tools for the differentiation of chromosomes and identification of structural chromosomal rearrangement. It facilitates the development of high-resolution karyotypes and detection of chromosomal variations in Arachis species. To our knowledge, the methodology presented in this study demonstrates for the first time the correlation between a sequenced chromosome region and a cytologically identified chromosome in peanut.
\end{abstract}

Keywords: Arachis species, Chromosome painting, Genomic evolution, High-resolution karyotype, Oligonucleotide multiplex

\section{Background}

Cultivated peanut (Arachis hypogaea L.) is an allotetraploid species that was evolved from a cross of two wild diploid progenitors, A. duranensis and A. ipaënsis [1]. It is widely cultivated worldwide as both an oil and cash crop. In 2016, total peanut production around the world was $43,982,063$ tons and average yield was $1710.49 \mathrm{~kg} \mathrm{ha}^{-1}$ [2]. However, peanut productivity is severely constrained by diseases and pests. The development of resistant cultivars is time-consuming and has been challenged by the existence of quantitative resistance traits and limited resistance resources in the cultivated gene pool [1].

The genus Arachis comprises 80 species [1], many of which potentially harbor beneficial genes for peanut improvement. Chromosome engineering is a useful approach by which genes from related species can be transferred and exploited. However, it is difficult to cytologically distinguish chromosomes of wild species because of their small size and the paucity of cytological markers. Therefore, many species, such as Arachis batizocoi, A. cruziana, A. krapovickasii, A. benensis, A. trinitensis, A. decora, A. palustris, and A. praecox have had incorrectly classified genomes [3, 4]. Staining with $4^{\prime}$, 6-diamidino-2-phenylindole (DAPI) and the use of rDNA probes are major tools that are traditionally used to differentiate peanut chromosomes [5-8]. The application of centromeric and telomeric repeat probes has significantly improved the ability to resolve Arachis karyotypes [9, 10]. Zhang et al. [11] identified seven $A$. duranensis bacterial artificial chromosome (BAC) clones that facilitated the differentiation of almost all chromosomes of peanut. Unfortunately, the existing markers were primarily distributed in the centromeric or telomeric regions with limited chromosomal polymorphism. In addition, the preparation of these markers is time-consuming and expensive.

SSONs, short DNA or RNA fragments, can be modified and directly hybridized with chromosomal DNA, thereby potentially replace traditional plasmid clones [12]. Oligopainting, using repetitive and single-copy sequences as probes, is a simple and efficient method that is being increasingly used in a wide variety of organisms, including humans [13], propionibacteria [14], cucumber [15], wheat
[16], and maize [17]. Two types of SSON probes are currently available: repetitive and single-copy. Repetitive SSONs can be derived from simple sequence repeats (SSRs), genomic sequence repeats (GSRs), and multi-copy genetic sequences (MCGSs) [18-21]. Single-copy SSONs include single-gene and single-copy genomic oligonucleotides [15, 22]. Repetitive SSONs, generally clustered together on one or several genomes or chromosomes, are frequently used to develop high-resolution karyotypes and identify chromosomes $[16,17,23]$. Single-copy SSONs are usually chromosome-specific, and are thus used to track specific chromosomes or chromosomal fragments and identify homoeologous chromosomes in closely related species $[13,15,24]$. In addition, SSONs can be used for species identification [25], haplotype analysis of homologous chromosomes [26], and RNA analysis of single cells [27, 28].

The availability of genome sequences of peanut and its donor species $A$. duranensis (A-genome) and A. ipaënsis (B-genome) [29], has facilitated the development of peanut SSON probes and provided a novel method by which peanut chromosomal structure and genomic evolution could be deciphered. The aim of the present study was to investigate chromosomal structural rearrangement and genome differentiation through high-resolution chromosome painting in the genus Arachis. The specific objectives were to: 1) develop repetitive SSON probes using $A$. duranensis genomic sequences, SSRs, and telomeric repeats; 2) utilize multiplex probes for simple and efficient chromosome identification; 3) compare high-resolution karyotypes of eight commercial cultivars, eight wild species, two interspecific $F_{1}$ hybrids, and one radiation-induced $M_{1}$ plant to reveal chromosomal rearrangements and genomic evolution; and 4) construct a chromosome-specific single-copy SSON library from the sequenced chromosome $6 \mathrm{~A}$ to identify the corresponding chromosome in the karyotype.

\section{Results}

\section{Development of repetitive SSON probes}

Eight repetitive SSON probes were identified and designed from different repetitive sequence sources. Probe sequences and the source of repetitive sequences are listed in Table 1. Sequential FISH/GISH was performed 
Table 1 Single-stranded oligonucleotide (SSON) probes developed in this study

\begin{tabular}{lll}
\hline SSON & Sequence and 5'- end modification & Origin \\
\hline DP-1 & TAMRA-5'-TGCGATCATACCAGCACTAATGCACCGGATCCCGTCAGAACTCTGAAGTTAAGCGTG - 3' & 5S rDNA (GenBank: M10470.1) \\
DP-2 & FAM-5'-ACTACTACTACTACTACTACTACTACTACT-3' & (ACT)10 \\
DP-3 & FAM-5'- CATTAAATCAGTTATAGTTTGTTGATGGTA-3' & Genomic DNA \\
DP-3 & TAMRA -5'- CATTAAATCAGTTATAGTTTTTTGATGGTA-3' & Genomic DNA \\
DP-4 & TAMRA-5'-ATTATTATTATTATTATTATTATTATTATT-3' & (ATT)10 \\
DP-5 & FAM-5'-TTTAGGGTTTAGGGTTAAGGGTTTAGGGTTTAGGGTTAGGGTTTAGGGTTTAGGG-3' & Telomere repeats \\
DP-6 & TAMRA-5'-AAAAAATCGGAGGAGCCTGCCGAAGATGAGG-3' & Genomic DNA \\
DP-7 & FAM -5'-AAAAAAAAAAAAAAAAAAAAAAAAAAAAAAA-3' & Genomic DNA \\
DP-7 & TAMRA-5'-AAAAAAAAAAAAAAAAAAAAAAAAAAAAAAA-3' & Genomic DNA \\
DP-8 & FAM -5'-TGAAAACTTTTATTTTTAAATTTTGAAACT-3' & Genomic DNA \\
DP-8 & TAMRA-5'-TGAAAACTTTTATTTTAAATTTTGAAACT-3' & Genomic DNA
\end{tabular}

to position these SSONs on chromosomes of Silihong (SLH), A. duranensis (the A-genome donor), and $A$. ipaënsis (the B-genome donor). All repetitive SSON probes generated distinguishable, stable, and clear signals on chromosomes of SLH, A. duranensis, and $A$. ipaënsis (Fig. 1 and Additional file 1: Figure S1 Additional file 2: Figure S2, Additional file 3: Figure S3, Additional file 4: Figure S4). The DP-1 probe produced signals that completely overlapped with those produced by $5 \mathrm{~S}$ rDNA plasmid clones in SLH (Fig. 1 and Additional file 1: Figure S1). Signals from DP-5 were distributed at the chromosome ends and in the centromeric, pericentromeric, and intercalary regions. They particularly formed larger clusters of strong signals in the long arm of $\mathrm{A}^{\text {hy }} 5$ and the short arm of $\mathrm{A}^{\text {hy }} 7$ of the A-genome, and in the centromeric region of $\mathrm{B}^{\text {hy }} 5$ of the B-genome (Fig. 1; Additional file 3: Figure S3). The DP-6 probe produced signals in 13 chromosomes of SLH. With the exception of one signal, which was detected on the long arm of chromosome $\mathrm{A}^{\text {hy }} 1$, all other signals were found in the centromeric regions of A- and B-genome chromosomes with various intensities. Most of the clear

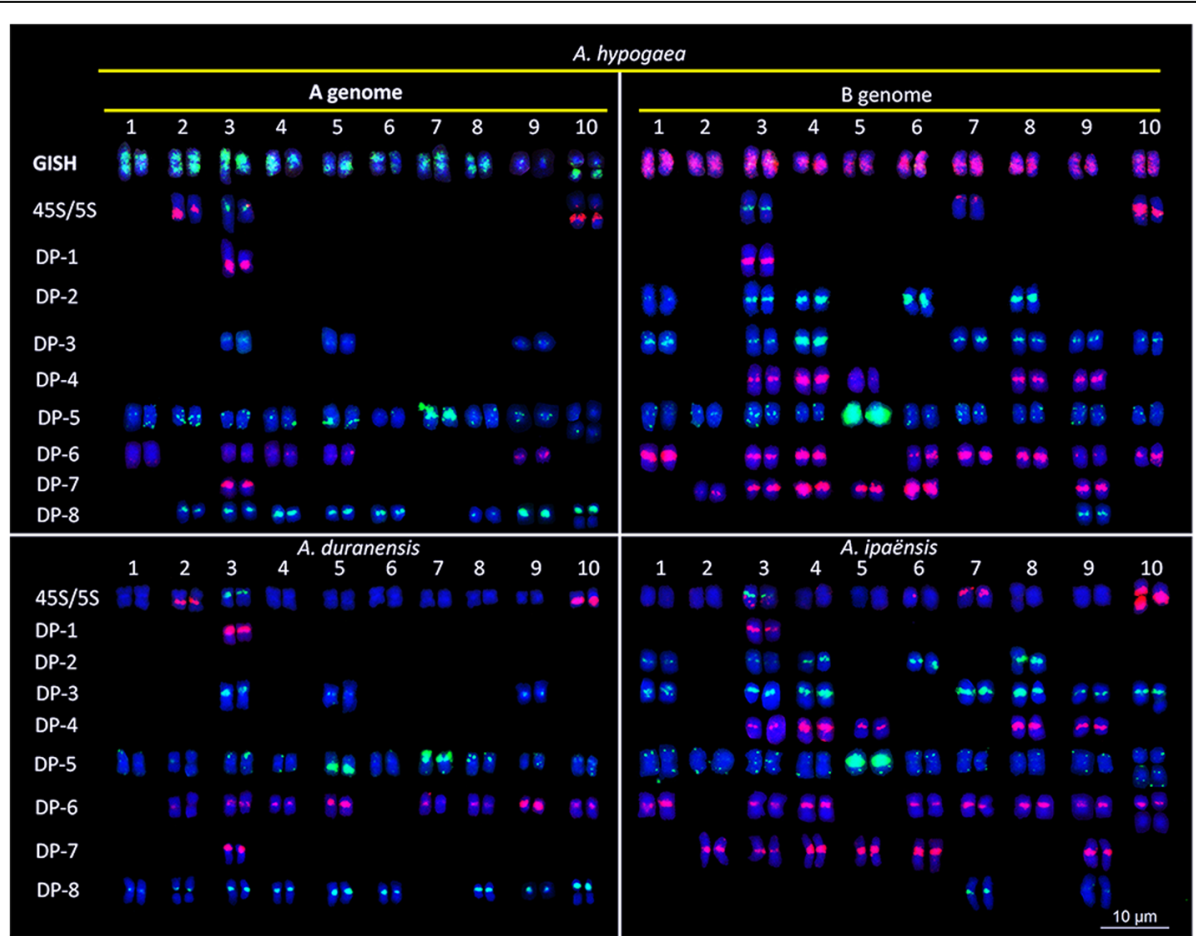

Fig. 1 FISH mapping of DP-1 (red), DP-2 (green), DP-3 (green), DP-4 (red), DP-5 (green), DP-6 (red), DP-7 (red), and DP-8 (green) by sequential FISH using $45 \mathrm{~S}$ rDNA (red), $5 \mathrm{~S}$ rDNA (green), and A. duranensis (green) and A. ipaënsis (red) total genomic DNA as probes in Arachis hypogea cv. $\mathrm{SLH}, \mathrm{A}$. duranensis, and A. ipaënsis. Blue color represents chromosomes counterstained with DAPI 
signals of DP-2, DP-3, DP-4, and DP-7 were observed only in the centromeric regions of B-genome chromosomes (Fig. 1). However, one exception was observed for DP-7, which revealed a clear signal in chromosome A3 near the 5S rDNA region, whereas an absence of, or sporadically weak signals were detected on the other A-genome chromosomes. In contrast to the four former SSONs, DP-8 only produced signals on eight A-genome chromosomes and on one B-genome chromosome in SLH (Fig. 1 and Additional file 4: Figure S4).

The eight SSON probes produced almost identical signals between the $\mathrm{B}^{\mathrm{i}}$ - and $\mathrm{B}^{\text {hy }}$-genome chromosomes of the wild donor A. ipaënsis and SLH. The only difference was that DP-8 produced a signal only on chromosome $\mathrm{B}^{\text {hy }} 9$ of SLH, but produced an additional signal on $\mathrm{B}^{\mathrm{i}} 7$ in A. ipaënsis. Most signals generated by these eight probes were also similar between the A-genome chromosomes of $A$. duranensis and SLH, with only few exceptions. For instance, the signal of DP- 5 at the interstitial position on the long arm of $\mathrm{A}^{\text {hy }} 5$ was weaker than that on $\mathrm{A}^{\mathrm{du}} 5$. The DP-6 probe produced signals at centromeric positions on the chromosomes of both species (A3, A4, A5, and A9), and other signals in SLH only ( ${ }^{\text {hy }} 1$ ), or A. duranensis only ( $\mathrm{A}^{\mathrm{du}} 2$, $\mathrm{Adu} 7, \mathrm{~A}^{\mathrm{du}} 8$, and $\left.\mathrm{A}^{\mathrm{du}} 10\right)$, or produced no signal at all $\left(\mathrm{A}^{\mathrm{du}} 1\right)$.

\section{High-resolution karyotyping of peanut based on} oligopainting using a repetitive SSON multiplex probe A multiplex \#1 repetitive SSON probe was developed according to the signal patterns of seven repetitive SSON probes that included 6-carboxyfluorescein (FAM) modified DP-2, 5, and 7, and 6-carboxytetramethylrhodamine (TAMRA) modified DP-1, 3, 4, and 6 (Table 2). Sequential FISH/GISH using Multiplex \#1, total genomic DNA of A. duranensis and A. ipaënsis, and 45S and 5S rDNA probes facilitated the development of a high-resolution karyotype for SLH (Additional file 5: Figure S5).

Table 2 Single-stranded oligonucleotide (SSON) multiplexes developed in this study

\begin{tabular}{lll}
\hline Multiplex & SSON with $5^{\prime}$ - end modified & Concentration $(\mathrm{ng} / \mathrm{\mu L})$ \\
\hline$\# 1$ & FAM - DP-2 & 100 \\
& FAM - DP-5 & 100 \\
& FAM - DP-7 & 26.67 \\
& TAMRA - DP-1 & 33.33 \\
& TAMRA - DP-3 & 100.00 \\
& TAMRA - DP-4 & 100.00 \\
& TAMRA - DP-6 & 100.00 \\
& FAM - DP-2 & 100 \\
& FAM - DP-5 & 100 \\
& FAM - DP-7 & 26.67 \\
& TAMRA - DP-8 & 26.67 \\
\hline
\end{tabular}

Significant differences in $\mathrm{A}^{\text {hy }}$ and $\mathrm{B}^{\text {hy }}$ chromosomes were visible in this karyotype. Among $\mathrm{B}^{\text {hy }}$ chromosomes, $\mathrm{B}^{\text {hy }} 5$ displayed distinct green centromeric signals, whereas $\mathrm{B}^{\text {hy }} 8$ and $\mathrm{B}^{\text {hy }} 10$ exhibited strong red centromeric signals. Both red and green signals were detected in the centromeric regions of all remaining chromosomes, with the exception of $\mathrm{B}^{\text {hy }} 2$, which displayed no signals. Strong signals of both colors were detected on $\mathrm{B}^{\text {hy }} 3, \mathrm{~B}^{\text {hy }} 4, \mathrm{~B}^{\text {hy }} 6$, and $B^{\text {hy }} 9$, whereas weaker signals of both colors were detected on $\mathrm{B}^{\text {hy }} 1$, and only strong red signal was detected on $B^{\text {hy }} 7$. Signals of $45 \mathrm{~S}$ or $5 \mathrm{~S}$ rDNA were detected on $\mathrm{B}^{\text {hy }} 3, \mathrm{~B}^{\text {hy }} 7$, and $\mathrm{B}^{\text {hy }} 10$. Among the $\mathrm{A}^{\text {hy }}$ chromosomes, $\mathrm{A}^{\text {hy }} 7$ and $\mathrm{A}^{\text {hy }} 5$, quite unlike the other $\mathrm{A}^{\text {hy }}$ chromosomes, showed strong interstitial telomeric signals, on the short arm of $\mathrm{A}^{\text {hy }} 7$ and on the long arm of $\mathrm{A}^{\text {hy }} 5$. Signals associated with both $45 \mathrm{~S}$ and $5 \mathrm{~S}$ rDNA loci were detected on $\mathrm{A}^{\text {hy }} 2, \mathrm{~A}^{\text {hy }} 3$, and $\mathrm{A}^{\text {hy }} 10$. Furthermore, $\mathrm{A}^{\text {hy }} 6$ displayed no signals, $\mathrm{A}^{\text {hy }} 4$ and $\mathrm{A}^{\text {hy }} 8$ displayed large green centromeric signals, and small red signals were detected on the long arm of $\mathrm{A}^{\text {hy }} 1$ (Fig. 2, Additional file 5: Figure S5 and Additional file 6: Figure S6).

To validate the potential of Multiplex \#1, seven other cultivars (N686, N607, N655, N734, N477, N587, and N419) were analyzed. In general, almost all chromosomes of these cultivars were similar to those of SLH, and the karyotypes of all cultivars remained conservative, with the exception of chromosomes $A^{\text {hy }} 10$ and $B^{\text {hy }} 1$, both of which showed minor variations in the centromeric and $45 \mathrm{~S}$ rDNA regions of different cultivars (Fig. 2).

\section{Chromosomal structural rearrangements revealed in wild Arachis species}

To assay genomic differentiation and chromosomal rearrangements, the chromosomes of eight wild species were discriminated by sequential FISH/GISH using Multiplex \#1 (Additional file 6: Figure S6 and Additional file 7: Figure S7). High-resolution karyotypes (Fig. 3) and idiograms of these species (Additional file 8: Figure S8) were developed and compared. Among these species, the genome of $A$. duranensis and A. ipaënsis showed a high similarity to the respective A and B genomes of SLH; however, a greater number of green signals were observed on the long arm of $\mathrm{A}^{\mathrm{du}} 5$ than on $\mathrm{A}^{\text {hy }} 5$ (Fig. 3 and Additional file 8: Figure S8).

Significant differences were observed on chromosomes $1,4,5,6,7,8,9$, and 10 of six A-genome species (SLH, A. duranensis, A. chiquitana, A. stenosperma, A. diogoi, and $A$. villosa). Signals were absent from chromosomes $A^{\text {hy }} 1, A^{d u} 1, A^{s} 6, A^{v} 6$, and $A^{\text {du }} 6$, whereas green signals were present on the other chromosomes. Chromosomes $\mathrm{A}^{\text {hy }} 4, \mathrm{~A}^{\mathrm{du}} 4, \mathrm{~A}^{\mathrm{v}} 4, \mathrm{~A}^{\text {hy }} 8, \mathrm{~A}^{\mathrm{du}} 8, \mathrm{~A}^{\mathrm{v}} 8, \mathrm{~A}^{\mathrm{s}} 8$, and $\mathrm{A}^{\mathrm{c}} 8$ exhibited green signals only, whereas $\mathrm{A}^{\mathrm{c}} 4, \mathrm{~A}^{\mathrm{s}} 4, \mathrm{~A}^{\mathrm{di}} 4$, and $\mathrm{A}^{\mathrm{di}} 8$ displayed both red and green signals. With the exception of the much larger chromosome, $\mathrm{A}^{\mathrm{c}} 9$, which exhibited only 


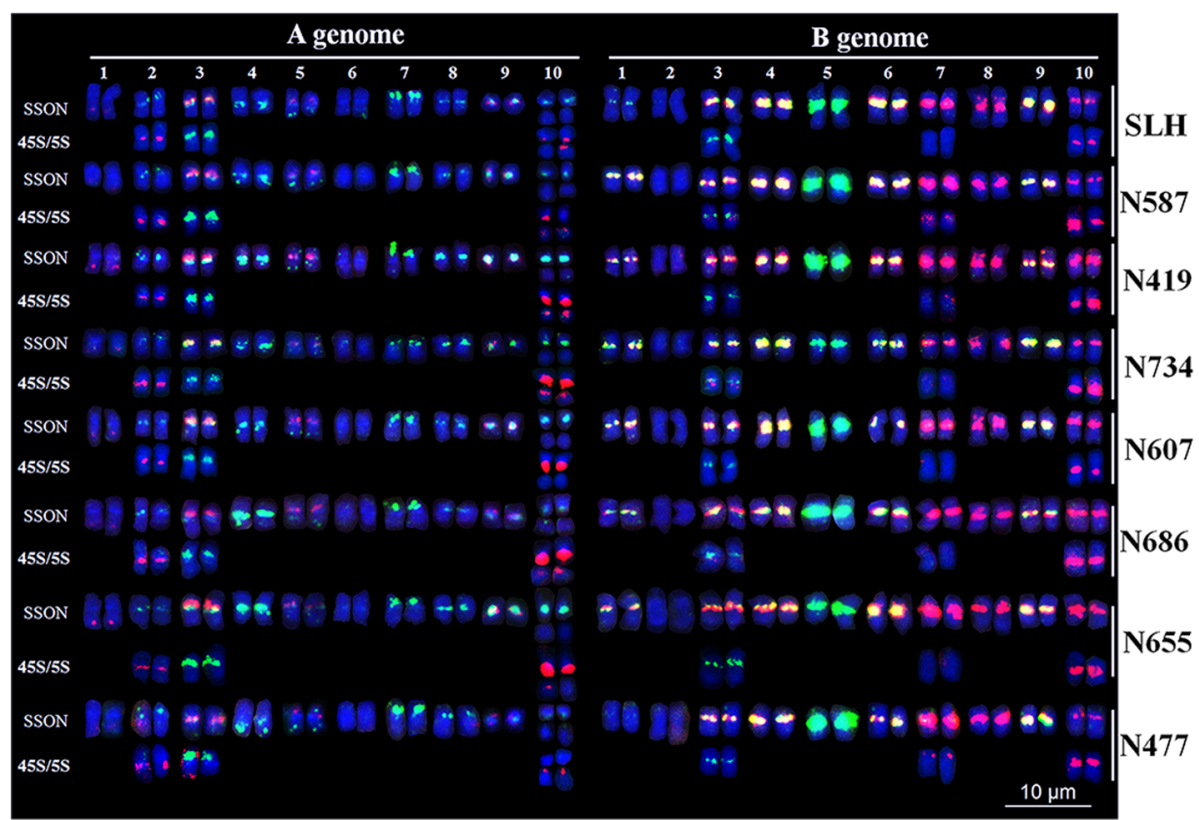

Fig. 2 Karyotypes of eight peanut varieties. Lines labeled as SSON and 45S/5S refer to signals from Multiplex \#1 and rDNAs, respectively. Blue color represents chromosomes counterstained with DAPI. In SSON, red represents signals of DP-1, DP-3, DP-4, and DP-6; and green represents signals of DP-2, DP-5, and DP-7. In 45S/5S, red represents signals of 45S rDNA and green represents signals of $5 \mathrm{~S}$ rDNA

green signals, chromosomes A9 among the other species were similar in size and displayed both green and red signals (Fig. 3 and Additional file 8: Figure S8). Green signals were absent from the long arm of chromosomes $\mathrm{A}^{\mathrm{s}} 5$ and $\mathrm{A}^{\mathrm{c}} 5$, and red signals were absent from the centromere of $\mathrm{A}^{\mathrm{c}} 5$. On chromosomes $\mathrm{A}$, green signals were detected at the ends of the short arms of $\mathrm{A}^{\text {hy }} 7$ and
$\mathrm{A}^{\mathrm{du}} 7$, the end of the long arm of $\mathrm{A}^{\mathrm{c}} 7$, and in the centromeric region of $A^{v} 7$. Red signals were observed on chromosome $\mathrm{A}^{\mathrm{s}} 10$ at the centromere. Based on these signal patterns, the genome of $A$. duranensis was evidently most similar to the $\mathrm{A}^{\text {hy }}$ chromosomes of SLH, followed by $A$. villosa, A. stenosperm, A. diogoi, and $A$. chiquitana (Fig. 3 and Additional file 8: Figure S8).

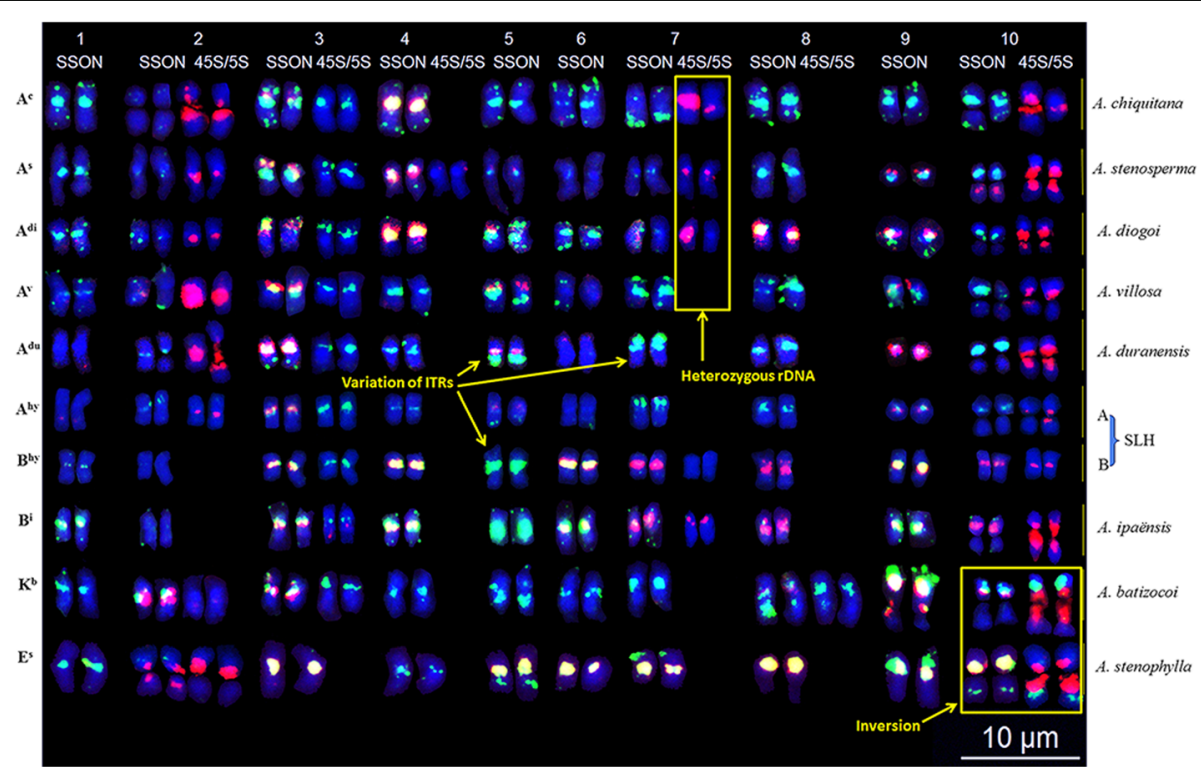

Fig. 3 Karyotypes of nine Arachis species. Columns labeled as SSON and 45S/5S refer to signals from Multiplex \#1 and rDNAs, respectively. Probe color same as Fig. 2 
Karyotypes of A. batizocoi and A. stenophylla accessions were significantly different from the A and B genomes. In both species, green signals covered almost the entire short arm of chromosome 9 , and $45 \mathrm{~S}$ and $5 \mathrm{~S}$ rDNA loci were detected on chromosome 10. The chromosomes of $A$. batizocoi and A. stenophylla were quite different from each other; $A$. batizocoi displayed $5 \mathrm{~S}$ rDNA signals on $\mathrm{K}^{\mathrm{b}} 3$ and $\mathrm{K}^{\mathrm{b}} 8$, whereas $A$. stenophylla did not; $E^{S} 5, E^{S} 6$, and $E^{S_{7}} 7$ exhibited both red and green signals in their centromeric regions, whereas only green signals were observed in these regions on $\mathrm{K}^{\mathrm{b}} 5, \mathrm{~K}^{\mathrm{b}} 6$, and $\mathrm{K}^{\mathrm{b}} 7$. Furthermore, the distribution of $45 \mathrm{~S}$ and $5 \mathrm{~S}$ rDNA loci showed opposite trends between $\mathrm{K}^{\mathrm{b}} 10$ and $\mathrm{E}^{\mathrm{S}} 10$, suggesting an inversion in one of the two species (Fig. 3 and Additional file 8: Figure S8).

Comparative analysis of all karyotypes revealed three types of structural rearrangements in the wild species studied. First, heterogeneous rDNA loci were found in different homologous chromosome pairs. For example, $45 \mathrm{~S}$ rDNA signals differed between the homologous chromosomes 7 in species of the A-genome. In particular, the intensity of the $45 \mathrm{~S}$ rDNA signals were identical in $A^{\mathrm{s}} 7$ and differed in $\mathrm{A}^{\mathrm{c}} 7$. The $45 \mathrm{~S}$ rDNA signal was detected only on the $\mathrm{A}^{\mathrm{di}} 7$ chromosome; however, these signals were lacking on $\mathrm{A}^{\mathrm{du}} 7$ (Fig. 3 and Additional file 8: Figure S8). Second, interstitial telomeric signal positions and amplifications were variable. Signals of different intensities and sizes were found at the chromosome ends $\left(A^{\mathrm{du}} 7\right.$ and $\left.E^{\mathrm{s}} 9\right)$, in the middle of chromosome arms $\left(A^{\text {du }} 5\right)$, or near centromeres (B $\left.{ }^{\text {hy }} 5\right)$ (Fig. 3 and Additional file 8: Figure S8). Third, chromosome morphology was variable among different species. For example, chromosomes $A^{c} 9$ and $A^{s} 9$ were small and metacentric; whereas $E^{s} 9$ was submetacentric and $E^{s} 8$ was subtelocentric (Additional file 8: Figure S8).

\section{Chromosomal characterization of interspecific $F_{1}$ hybrids and an irradiation-induced $M_{1}$ plant}

To further validate the potential of Multiplex \#1, two interspecific $F_{1}$ hybrids were examined by sequential FISH/GISH. Two genome sets of the hybrid w1510 were identical to $\mathrm{A}^{\text {hy }}$ and $\mathrm{B}^{\text {hy }}$ of $\mathrm{SLH}$, and the third genome set was the same as $\mathrm{K}^{\mathrm{b}}$ of $A$. batizocoi (Fig. 4 and Additional file 9: Figure S9). For w1612, two genome sets were identical to $\mathrm{A}^{\text {hy }}$ and $\mathrm{B}^{\text {hy }}$ of N734, and the third was similar to $\mathrm{E}^{\mathrm{S}}$ of $A$. stenophylla (Fig. 4 and Additional file 9: Figure S9). These results indicate that both w1510 and w1612 were true $F_{1}$ hybrids.

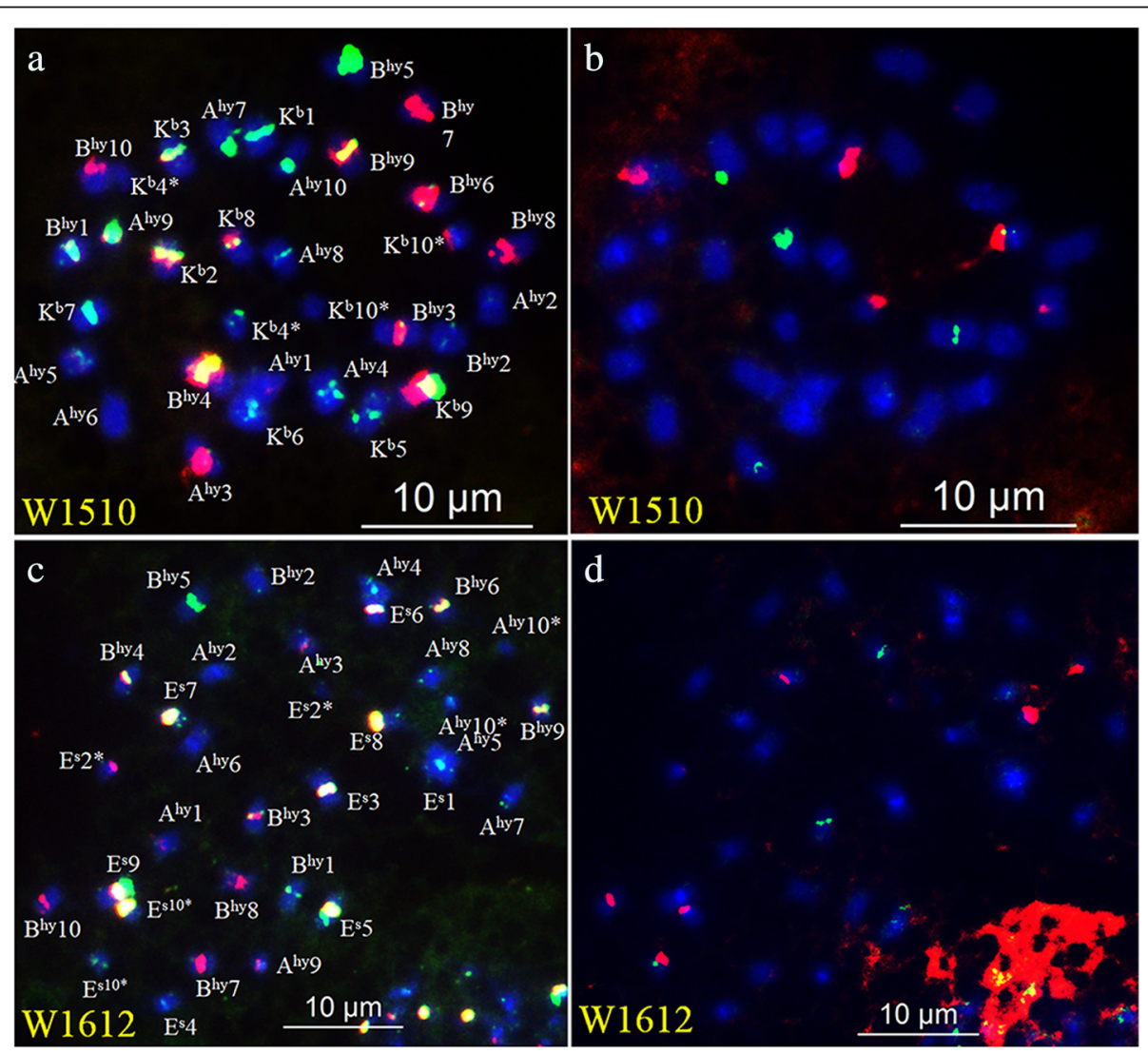

Fig. 4 Sequential FISH of two hybrids $F_{1}$ w1510 and w1612 using Multiplex \#1 (a and c), and 5S and 45S rDNA (b and d) plasmid clones as probes. a-b: w1510 derived from SLH (A. hypogaea) and A. batizocoi; c-d: w1612 derived from N734 (A. hypogaea) and A. stenophylla. Probe color as Fig. 2 
Multiplex \#2, which included TAMRA-modified DP-8, and FAM-modified DP-2, DP-5, and DP-7, was used for oligopainting with sequential GISH/FISH to characterize a radiation-induced $\mathrm{M}_{1}$ plant (162-30). By applying this probe, we were able to identify two chromosomal translocations and one monosomic chromosome (Fig. 5a 5f). One translocation between $\mathrm{A}^{\text {hy }} 4$ and $\mathrm{B}^{\text {hy }} 4$ that contained two centromeric DAPI bands and SSON centromeric bands of both colors, formed a potential dicentric chromosome T4BL-4BS-4AS-4AL. The other translocation, T10BS-10AS.10AL, involved a large satellite. The monosomic chromosome was identified in the $\mathrm{B}^{\text {hy }} 3$ chromosome because of its unique signal patterns (Fig. $5 \mathrm{~g}$ and $\mathrm{h}$ ).

\section{Oligopainting using the single-copy sequence library of chromosome $6 \mathrm{~A}$ as probes, decorated the cytologically designated chromosome $A 3$ of $A$. duranensis}

To correlate a sequenced chromosome with a cytologically identified chromosome, a single-copy SSON library was developed from a $\sim 8.5 \mathrm{Mb}$ distal region of $6 \mathrm{~A}$ and used to paint metaphase chromosomes of A. duranensis. A pair of clear signals was reputably detected on one pair of chromosomes of A. duranensis (Additional file 10: Figure S10). Sequential FISH using 45S, $5 \mathrm{~S}$ rDNA, and DP-5 probes (Additional file 11: Figure S11) revealed that the target signals were present in cytologically designated chromosome A3, which displayed a typical distribution of $5 \mathrm{~S}$ rDNA on its short arm (Fig. 6).

\section{Discussion}

Oligopainting using repetitive and single-copy sequences as probes provides a simple and efficient method for chromosome identification in peanut

Chromosome identification has previously been carried out in peanut using primarily FISH with $45 \mathrm{~S}$ and $5 \mathrm{~S}$ rDNA probes and DAPI staining [6-8]. Zhang et al. [11] recently developed seven BAC clones in Arachis as powerful new markers for chromosome identification. In the present study, eight repetitive SSON probes and one single-copy SSON library were successfully developed, and the repetitive SSON probes were combined into two powerful multiplexes (Multiplex \#1 and Multiplex \#2) for efficient chromosome discrimination. The two multiplexes effectively differentiated the chromosomes and genomes of eight peanut cultivars, eight wild species, two interspecific hybrids, and one radiation-induced $\mathrm{M}_{1}$ plant, and revealed structural rearrangements in these species and accessions. Repetitive SSON probes for the identification of peanut chromosomes can be easily designed and modified, thus greatly reducing the required time and expense. The repetitive SSON probes can be used advantageously in nondenaturing FISH, thereby further simplifying the FISH procedure [16]. Our findings demonstrated that repetitive SSON probes could be useful and provide powerful tools for peanut chromosome engineering. However, the current SSONs did not cover all chromosome regions and involved only limited types of repetitive sequences. A further study should utilize various sources of repetitive sequences to identify specific chromosomes or regions.

The International Peanut Sequencing Project has sequenced the reference genomes of $A$. duranensis and $A$. ipaënsis [29]. However, no direct correspondence has been established between the peanut linkage map and cytologically identified chromosomes. Based on the genome sequence of chromosome A6 of $A$. duranensis, we successfully developed a chromosome-specific single-copy SSON library, which could be used to paint the target chromosomes. By using both the repetitive SSONs and single-copy sequences as probes for oligopainting, we identified the target chromosomes and successfully correlated a sequenced A6 segment to the actual chromosome. This opens a path to further correlation of all sequenced chromosomes with cytologically identified chromosomes. The development of more single-copy SSON libraries for each chromosome of peanut is underway at our institute.

\section{New clues for genomic evolution revealed by painting of Arachis species}

In the cultivated peanut and its wild ancestors, chromosome painting with the eight probes generated fewer and weaker signals on A-genome chromosomes than on those of the B-genome. For example, three SSON probes, DP-2, -4 , and -7 , derived from SSRs $(\mathrm{ACT})_{10}$, $(\mathrm{ATT})_{10}$, and $(\mathrm{A})_{31}$, respectively, rarely produced signals on A-genome chromosomes, but yielded strong signals on chromosomes of the B-genome, which showed more SSRs variations in A. ipaënsis than in A. duranensis. These results are consistent with the fact that although mono-, di-, and tri-nucleotides are the most abundant motifs in A. duranensis and A. ipaënsis, a greater number of SSRs are present in A. ipaënsis than in A. duranensis [30]. In addition, A. ipaënsis contains more transposable elements than $A$. duranensis does [29]. This suggests that the B-genome has more repeats, and may explain why the size of B-genome is larger than that of the A-genome (1.56 Gb vs $1.25 \mathrm{~Gb})$ [29]. Other repetitive SSONs also produced weaker and fewer signals on A chromosomes, in comparison to B chromosomes. For example, signals between $\mathrm{B}^{\mathrm{i}}$ and $\mathrm{B}^{\text {hy }}$ genomes, with the exception of DP-8 on chromosome 7, were almost identical in terms of position and intensity; however, fewer signals were present on four chromosomes of the $A^{\text {hy }}$ genome relative to $A^{\text {du }}$. Moreover, some signals on the $A^{\text {hy }}$ genome, such as $A^{\text {hy }} 5$, varied according to their quantity and position (Fig. 1), suggesting that during evolution, large chromosomal rearrangements occurred in the A-genome chromosomes rather than 


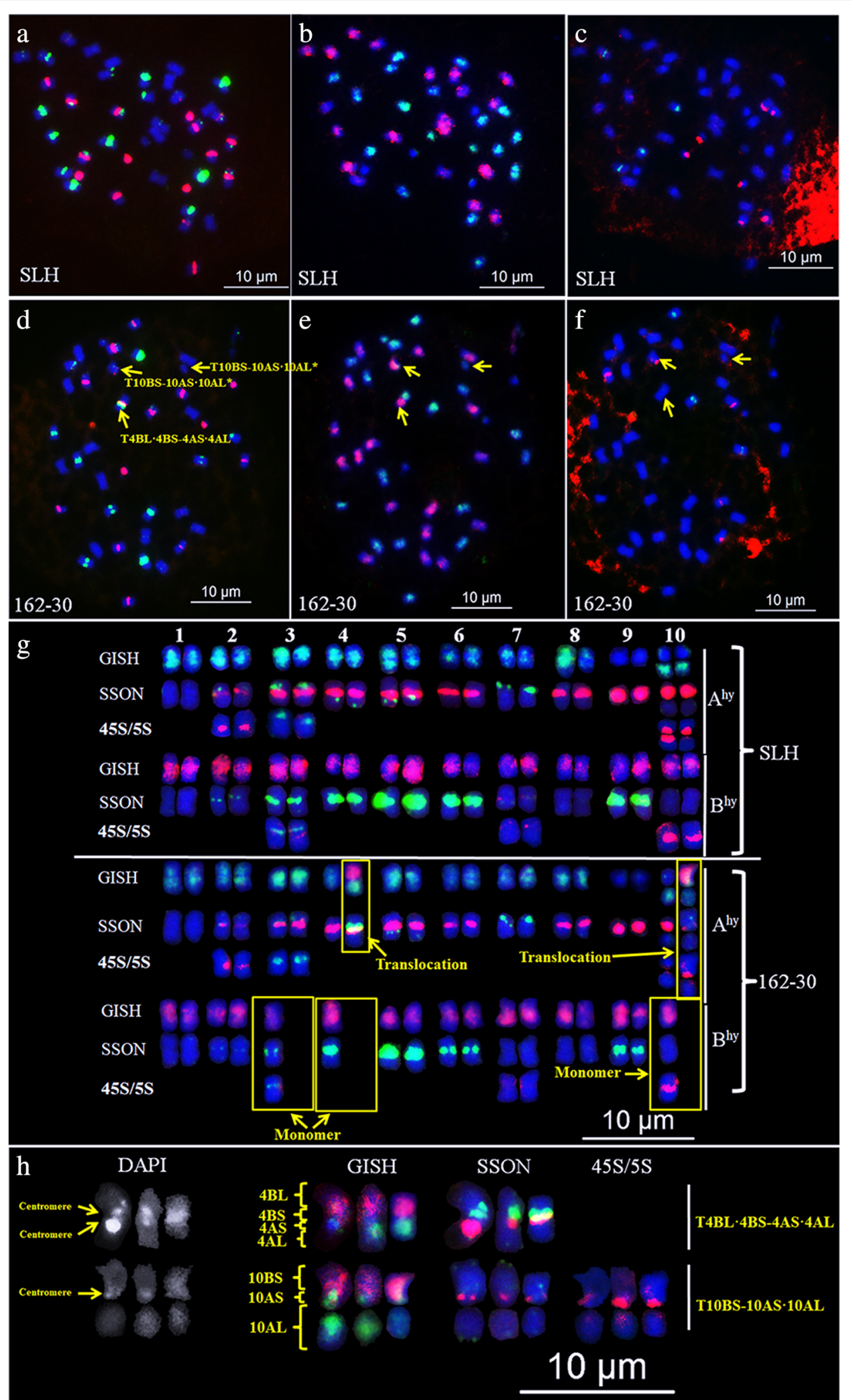

Fig. 5 (See legend on next page.) 
(See figure on previous page.)

Fig. 5 Chromosome aberrations detected in radiation-induced $M_{1}$ plant 162-30 of peanut cultivar SLH after sequential FISH/GISH. a-f Results of sequential FISH/GISH in SLH (a-c) and M1 plant 162-30 (d- $\mathbf{f})$ using Multiplex \#2 (a and d), A. duranensis genomic DNA (b and e; green), A. ipaënsis genomic DNA (b and $\mathbf{e}$; red), $5 \mathrm{~S}$ rDNA (c and $\mathbf{f}$; green), and 45S rDNA (c and f; red). g Karyotypes of SLH and 162-30. $\mathbf{h}$ Translocated chromosomes in 162-30. Multiplex \#2 contains four SSONs, including TAMRA-DP-8, FAM-DP-2, FAM-DP-5, and FAM-DP-7

in the B-genome chromosomes. These results correspond well with the findings of Bertioli et al. [29] and Zhang et al. [11].

Although the eight tested cultivars represented three different peanut taxa (varieties. Fastigiata, hypogea, and vulgaris), their karyotypes were similar to each other, even though a signal variation was found between chromosomes $\mathrm{A}^{\text {hy }} 10$ and $\mathrm{B}^{\text {hy }} 1$. This observation provides evidence that these cultivated peanut varieties arose from a single allotetraploid species [31].

Our results indicated that the genome of $A$. duranensis was most similar to the A-genome of the cultivated peanut, followed by the genomes of $A$. villosa, A. stenosperma, A. diogoi, and A. chiquitana, supporting the contention that A. duranensis was the donor of the A-genome [7]. Similarly, the identical karyotypes observed between $A$. ipaënsis and the B-genome of cultivated peanut supports the notion of A. ipaënsis as the B-genome donor [3, 8, 29, 32].

Chromosome identification by oligopainting likewise facilitates the classification of species. A. chiquitana was originally grouped with $\mathrm{P}$ genome species [33]. However, because it carried a small "A chromosome" and showed similar 45S rDNA, 5S rDNA, and DAPI banding patterns to those of A-genome species, it was regrouped with species of the A-genome [7]. In the present study, the karyotype of A. chiquitana was considerably different from
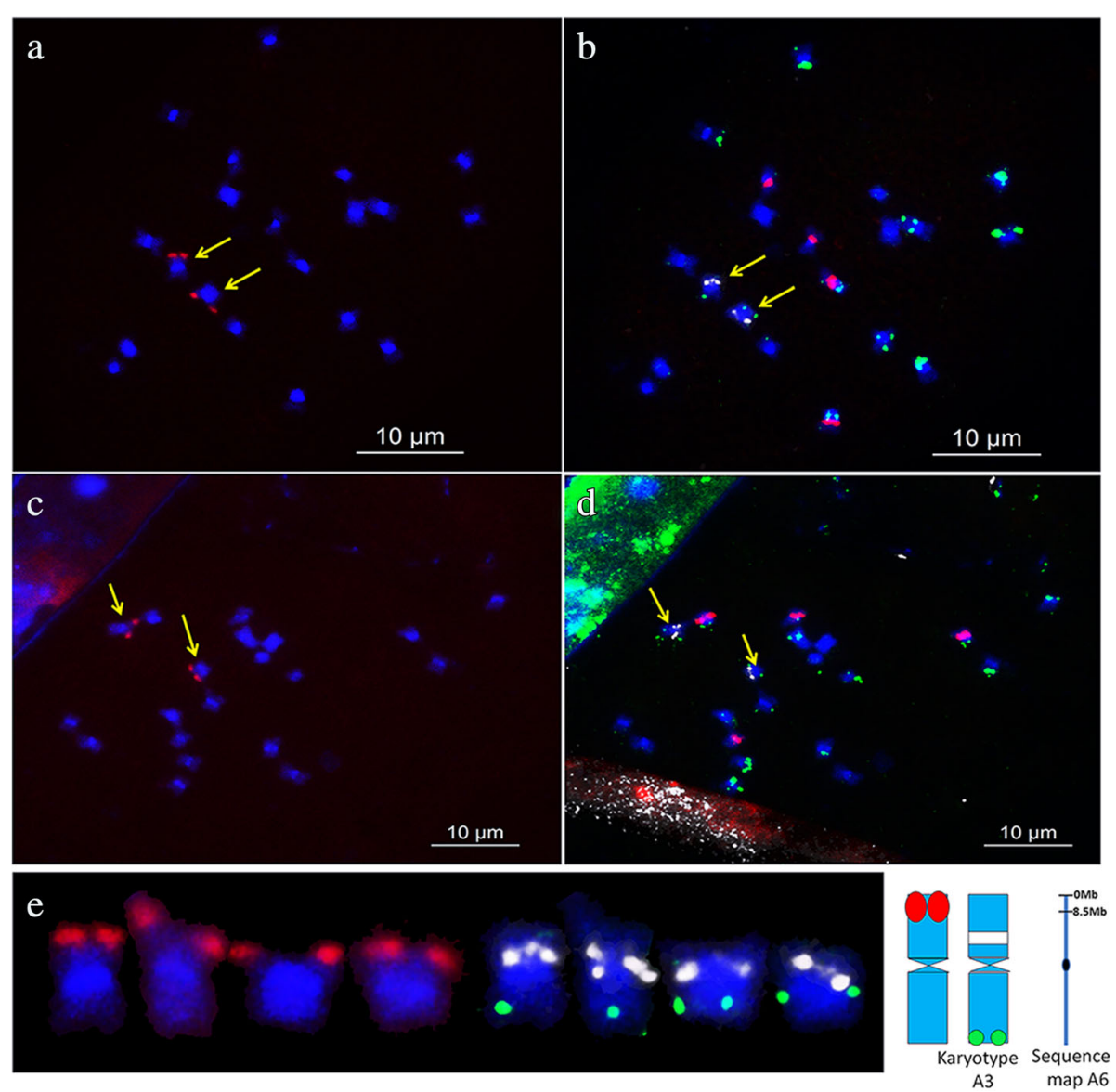

Fig. 6 Oligopainting using single-copy oligonucleotide library 6A-1 combined with sequential FISH using DP-5, and plasmid clones 45S rDNA and $5 \mathrm{~S}$ rDNA as probes in A. duranensis. Blue color represents chromosomes counterstained with DAPl; a and $\mathbf{c}$ shows oligopainting using single-copy SSON library 6A-1 (green); b and d, sequential FISH using repetitive SSON DP-5 (green), 45S rDNA (red), and 5S rDNA (white inverted from the original green); c From left to right: chromosome with signals of library 6A-1 (green); karyotype of A3, probe signals are same as (b) and (d); idiograms of the chromosome displaying signals from library 6A-1 and the karyotype of A3; and sequence map of A6 showing 0-8.5 Mb regions corresponding to library $6 \mathrm{~A}-1$. Yellow arrows in panels $(\mathbf{a}-\mathbf{d})$ indicate chromosomes with signals from library $6 \mathrm{~A}-1$ 
those of other species. For example, the morphology and repetitive SSON banding pattern of $\mathrm{A}^{\mathrm{c}} 9$ were very distinct from those of A9 chromosomes of other species with the A-genome. The genome of $A$. chiquitana thus does not seem to be associated with the species group bearing the A-genome. Genomes $E^{S}$ and $K^{b}$ are more closely related to each other than they are to other genomes, even though they belong to different sections of the genus Arachis.

Comparative karyotyping by SSON DP-5 revealed that variable internal telomeric repeats (ITRs) exist in chromosomes. They might have descended from telomere-mediated chromosomal rearrangements in these repeats during genomic evolution in peanut. Heterogeneous ITRs have also been observed in tomato, and some ITRs were evidently extremely amplified [34]. Chromosome 10 of A. stenophylla and A. batizocoi both contained an obvious inversion in the region containing $45 \mathrm{~S}$ and $5 \mathrm{~S}$ rDNA loci, providing further evidence for chromosomal structural rearrangements.

From both our previous report [10] using the PCR-amplified telomeric repeats as probes, and the present results using multiple oligonucleotide probes, the discovery of heterogeneous distributions of rDNAs, chromosome inversions, duplications, and morphological variations in different peanut genomes has indicated that Arachis might have undergone significant chromosomal rearrangements during genome evolution. Further oligopainting using a greater number of repetitive and single-copy SSON probes should allow us to gain more information on these changes.

\section{Conclusions}

Repetitive and single-copy SSONs and multiplex repetitive SSON probes developed in the present study can be useful tools for simple and efficient chromosome identification and genomic differentiation, and facilitate chromosome engineering in peanut. Oligopainting using repetitive and single-copy sequences as probes showed that modern peanut cultivars have almost identical karyotypes, indicating low genetic diversity. In contrast, wild species exhibited an abundance of chromosomal rearrangements and variations, suggesting their genomic potential for peanut improvement. With the availability of refined reference genome sequences of peanut, additional repetitive and single-copy SSON probes can be developed and used to correlate genetic and cytological maps. Further chromosomal rearrangements, including translocations, inversions, and duplications, should be identifiable using these types of probes. The repetitive and single-copy SSON probes and methodology described in the present study provide useful tools for further application and cytogenetic study in peanut.

\section{Methods}

\section{Plant materials}

Eight accessions of cultivated peanut (A. hypogaea) ( $2 n$ $=2 x=40)$ and eight diploid Arachis species $(A$. chiquitana, A. stenosperma, A. diogoi, A. villosa, A. duranensis, A. ipaënsis, A. batizocoi, and A. stenophylla) were used in this study. Five cultivars (SLH, Kainong30, Linguidachezi, Yuhua15, Liuchengzhenzhudou) were from Henan Academy of Agricultural Sciences. The other three cultivars and eight diploid species were introduced from National Germplasm Resources Laboratory of USA (Beltsville, MD). Accession number, chromosome number, genomic constitution, origin and identification of each plant material are listed in Table 3. In addition, two interspecific $\mathrm{F}_{1}$ hybrids (w1510 from a cross between the SLH variety and $A$. batizocoi, and w1612 from a cross between the N734 variety and A. stenophylla), and one irradiation-induced SLH $\mathrm{M}_{1}$ plant (designated as 16230) generated by pollen irradiation at a dosage of $16 \mathrm{~Gy}$ using a ${ }^{60} \mathrm{Co}$ source (Isotope Institute Co. Ltd., Henan Academy of Sciences) were also used as plant materials.

\section{Chromosome preparation}

Seeds were germinated for $7 \mathrm{~d}$ on moist filter paper at $25{ }^{\circ} \mathrm{C}$. Healthy lateral root tips were excised and pretreated with $2 \mathrm{mM}$ 8-hydroxyquinoline for $3 \mathrm{~h}$ at $25^{\circ} \mathrm{C}$, fixed in 3:1 absolute ethanol: glacial acetic acid for $5 \mathrm{~h}$ at $25{ }^{\circ} \mathrm{C}$, and stored at $-20{ }^{\circ} \mathrm{C}$. Root meristem sections of $0.3-0.5 \mathrm{~mm}$ were excised, squashed in $45 \%$ glacial acetic acid, and frozen at $-80{ }^{\circ} \mathrm{C}$ for $12 \mathrm{~h}$. The chromosome spreads were dehydrated in $100 \%$ ethanol and air-dried after removal of the cover slips.

\section{Repetitive SSONs and single-copy SSON design and labeling}

Eight repetitive SSON probes, named DP-1 to DP-8, were selected from different sources of repetitive sequences. The DP-2 and DP-4 probes were screened from 10 tri-nucleotide SSRs, including (AAC) $)_{10}$, (AAG) $)_{10}$, $(\mathrm{AGG})_{10},(\mathrm{ACT})_{10},(\mathrm{CAT})_{10},(\mathrm{CAC})_{10},(\mathrm{ACG})_{10},(\mathrm{CAG})_{10}$, $(\mathrm{AAT})_{10}$, and $(\mathrm{GCC})_{10}$, and four di-nucleotide SSRs, namely $(\mathrm{AT})_{15},(\mathrm{GC})_{15},(\mathrm{AG})_{15}$, and $(\mathrm{AC})_{15}$, as described by Cuadrado et al. [18] and Du et al. [16] (Table 1). The design of the DP-5 probe was based on the repeat sequence (TTTAGGG) , as described by Cuadrado et al. [12] and Du et al. [10]. The DP-1 probe was designed by Blasting the wheat $5 \mathrm{~S}$ rDNA sequence against genomic sequences of $A$. duranensis (Table 1). Whereas DP-3, DP-6, DP-7, and DP-8 were designed following the method of $\mathrm{Du}$ et al. [16] using the $4 \mathrm{~Gb}$ genomic sequence of $A$. duranensis (unpublished data). The A. duranensis genomic sequence $k$-mers were counted using the Jellyfish tool (Version 1.1.11) [35] with the following parameters: $k$-mer $=31 \mathrm{nt}$ and copy number $>1000$. The 
Table 3 Plant materials used in this study

\begin{tabular}{|c|c|c|c|c|c|c|c|}
\hline \multirow{2}{*}{$\begin{array}{l}\text { Accession } \\
\text { number }\end{array}$} & \multirow{2}{*}{$\begin{array}{l}\text { PI number or } \\
\text { variety name }\end{array}$} & \multicolumn{2}{|l|}{ Identification } & \multirow[t]{2}{*}{ Origin } & \multirow[t]{2}{*}{ Section (genome) } & \multirow[t]{2}{*}{ Species (type) } & \multirow[t]{2}{*}{ Reference } \\
\hline & & Collector & No. & & & & \\
\hline$\overline{S L H}$ & Silihong & Zhang $X Y$ & & China & Arachis ( $A^{\text {hy }} A^{\text {hy }} B^{\text {hy }} B^{\text {hy }}$ ) & A. hypogaea (var. fastigiata) & \\
\hline N686 & PI 158854 & Holbrook & GP-134 & China & Arachis ( $A^{\text {hy }} A^{\text {hy }} B^{\text {hy }} B^{\text {hy }}$ ) & A. hypogaea (var. hypogaea) & [42] \\
\hline N607 & Kainong30 & Zhang $X Y$ & & China & Arachis ( $A^{\text {hy }} A^{\text {hy }} B^{\text {hy }} B^{\text {hy }}$ ) & A. hypogaea (var. hypogaea) & \\
\hline N655 & Linguidachezi & Zhang $X Y$ & & China & Arachis ( $A^{\text {hy }} A^{\text {hy }} B^{\text {hy }} B^{\text {hy }}$ ) & A. hypogaea (var. hypogaea) & \\
\hline N734 & Yuhua15 & Zhang $X Y$ & & China & Arachis ( $A^{\text {hy }} A^{\text {hy }} B^{\text {hy }} B^{\text {hy }}$ ) & A. hypogaea (var. hypogaea) & \\
\hline N477 & PI 240560 & Holbrook & GP-14 & South Africa & Arachis ( $A^{\text {hy }} A^{\text {hy }} B^{\text {hy }} B^{\text {hy }}$ ) & A. hypogaea(var. hypogaea) & {$[42]$} \\
\hline N587 & PI 290560 & Holbrook & GP-162 & India & Arachis ( $A^{\text {hy }} A^{\text {hy }} B^{\text {hy }} B^{\text {hy }}$ ) & A. hypogaea (var. fastigiata) & {$[42]$} \\
\hline N419 & Liuchengzhenzhudou & Zhang $X Y$ & & China & Arachis ( $A^{\text {hy }} A^{\text {hy }} B^{\text {hy }} B^{\text {hy }}$ ) & A. hypogaea (var. vulgaris) & \\
\hline Zw51 & PI 338280 & Hammons & 410 & Brazil & Arachis $\left(A^{5} A^{5}\right)$ & $\begin{array}{l}\text { A. stenosperma Krapov. \& } \\
\text { W. C. Greg. }\end{array}$ & {$[1,7,43]$} \\
\hline Zw52 & PI 298639 & Krapovickas & 9484 & Bolivia & Arachis $\left(K^{b} K^{b}\right)$ & $\begin{array}{l}\text { A. batizocoi Krapov. \& } \\
\text { W. C. Greg. }\end{array}$ & {$[3,43]$} \\
\hline Zw53 & PI 468322 & Krapovickas & 30,076 & Bolivia & Arachis $\left(B^{i} B^{i}\right)$ & $\begin{array}{l}\text { A. ipaensis Krapov. \& } \\
\text { W. C. Greg. }\end{array}$ & {$[3,43]$} \\
\hline Zw55 & PI 219823 & Krapovickas & 7988 & Argentina & Arachis $\left(A^{d u} A^{d u}\right)$ & $\begin{array}{l}\text { A. duranensis Krapov. \& } \\
\text { W. C. Greg. }\end{array}$ & {$[7,43]$} \\
\hline Zw56 & PI 298636 & Tweedi & 22,585 & Argentina & Arachis $\left(A^{v} A^{v}\right)$ & A. villosa Benth. & {$[1,7,43]$} \\
\hline Zw57 & PI 276235 & Simpson and Charles & 10,602 & Paraguay & Arachis $\left(A^{d i} A^{d i}\right)$ & A. diogoi Hoehne & {$[1,7,43]$} \\
\hline Zw59 & PI 476006 & Krapovickas & 36,027 & Bolivia & Arachis $\left(A^{C} A^{C}\right)$ & A. chiquitana Krapov. et al. & {$[7,43]$} \\
\hline Zw61 & PI 468178 & Gregory & 30,136 & Brazil & Erectoides $\left(\mathrm{E}^{\mathrm{s}} \mathrm{E}^{\mathrm{s}}\right)$ & $\begin{array}{l}\text { A. stenophylla Krapov. \& } \\
\text { W. C. Greg. }\end{array}$ & {$[1,43]$} \\
\hline
\end{tabular}

repeat sequences were analyzed by the CD-HIT Suite and DNAMAN software, and those sequences having more than $80 \%$ identity with other repeats were discarded. From the remaining 18,052 oligonucleotides, repetitive SSONs with the highest copy number were selected. This selection resulted in 150 repetitive SSONs that were synthesized and labeled by random labeling, and analyzed on SLH chromosomes. Four repetitive SSON probes, DP-3, DP-6, DP-7, and DP-8, were then preferably selected for use in the present study because of their stronger signals. All eight repetitive SSONs were modified at the 5 '-ends with TAMRA or FAM by the Invitrogen Company (Shanghai, China) and are listed in Table 1.

Single-copy SSONs were developed according to the method described by Han et al. [15], in which the genomic sequences of chromosome $6 \mathrm{~A}$ of $A$. duranensis in PeanutBase [36] were downloaded and analyzed. Using the distal $\sim 8.5 \mathrm{Mb}$ region of chromosome $6 \mathrm{~A}$ as a target region, all repetitive sequences were masked with RepeatMasker [37]. The remaining sequences were divided into single-copy SSONs of 42-48 nt in a stepwise size of $5 \mathrm{nt}$. Single-copy SSONs containing more than $6 \mathrm{nt}$ of homopolymers were discarded. Each single-copy SSON was aligned to the genomic reference sequence of A. duranensis using the BLAT tool [38] and screened for homologs with $>75 \%$ similarity in the genome. Melting point ${ }^{\mathrm{Tm}}$ and hairpin $\mathrm{Tm}$ values of each single-copy SSON were calculated using the Primer3 software [39]. Single-copy SSONs with $\mathrm{d} T m>10{ }^{\circ} \mathrm{C}(\mathrm{d} T m=T m-$ hairpin $\mathrm{Tm}$ ) were retained for probe construction. As a result, 20,000 selected single-copy SSONs were synthesized by MYcroarray (Ann Arbor, MI, USA). The resulting library was amplified and labeled with digoxigenin-11-dUTP according to the MYcroarry_MYtags labeling protocol [40].

\section{FISH and sequential FISH analysis}

Total genomic DNA was extracted from fresh young leaves of $A$. duranensis and A. ipaënsis [41]. Clones of 5S and $45 \mathrm{~S}$ rDNA of wheat (Triticum aestivum L.) were provided by Dr. Bikram S Gill, of Kansas State University, USA. The 5S rDNA clone and total genomic DNA of $A$. ipaënsis were labeled with biotin-16-dUTP (Roche) by nick translation and detected with fluorescein anti-biotin (Roche), whereas 45S rDNA and total genomic DNA of $A$. ipaënsis were labeled with digoxigenin-11-dUTP (Roche) and detected with anti-digoxigenin-rhodamine (Roche).

The FISH procedure followed the method described by Zhu et al. [17]. Briefly, the hybridization solution contained $7.5 \mu \mathrm{L}$ formamide (Sigma), $1.5 \mu \mathrm{L} 20 \times$ saline-sodium citrate (SSC) buffer, $1.0 \mu \mathrm{L}$ of each probe, $2.0 \mu \mathrm{L}$ of $50 \%$ dextran sulfate (Sigma), and $\mathrm{ddH}_{2} \mathrm{O}$ added to bring the total volume to $15 \mu \mathrm{L}$. The hybridization solution was denatured at $105{ }^{\circ} \mathrm{C}$ for $13 \mathrm{~min}$, and the 
spread chromosomes were denatured in $70 \%$ formamide at $75{ }^{\circ} \mathrm{C}$ for $70 \mathrm{~s}$, and then hybridized overnight at $37^{\circ} \mathrm{C}$. After hybridization, the slides were washed three times in $2 \times$ SSC at room temperature, and mounted with VECTASHIELD Mounting Medium, following which DAPI staining was performed.

Sequential FISH/GISH procedures were performed to map the signals of oligonucleotide probes. Briefly, slides were denatured after FISH using repetitive and single-copy SSON probes, and then washed to remove all signals, and subsequently dried. The GISH procedure was performed using total genomic DNA of A. duranensis and A. ipaënsis as probes to identify A- and B-genome chromosomes, respectively. A second FISH procedure using $45 \mathrm{~S}$ rDNA and $5 \mathrm{~S}$ rDNA probes was conducted on the same slides to develop karyotypes, as described by Du et al. [10].

\section{Fluorescence microscopy and imaging}

Slides were examined using a Leica DM6000 fluorescence microscope (Leica). Separate images from each filter set were captured using a cooled CCD camera (Leica). Images were optimized for contrast and brightness using Adobe Photoshop. To distinguish the signals produced in each chromosome by different probes after sequential FISH, the original green signal of $5 \mathrm{~S}$ rDNA was converted to pseudo-color white. For karyotyping and chromosome diversity analysis, 3-5 cells of each accession, including 6-10 samples of each chromosome, were observed. Most karyotypes were developed from a single cell, unless they were derived from overlapping chromosomes. Chromosomes of each species were primarily ordered based on morphology, size, and unique patterns, as reported by Du et al. [10].

\section{Additional files}

Additional file 1: Figure S1. FISH mapping of DP-2 (b, g, and l; green) and DP-1 ( $c, h$, and $m$; red) by sequential FISH using $45 \mathrm{~S}$ rDNA ( $\mathrm{e}, \mathrm{g}$ and $\mathrm{j}$; red), $5 \mathrm{~S}$ rDNA (e, g and $\mathrm{j}$, green), and $A$. duranensis (a, green) and $A$. ipaënsis (a, red) total genomic DNA as probes in SLH (a-e), A. duranensis $(f-j)$, and A. ipaënsis (k-o). (TIF $2370 \mathrm{~kb}$ )

Additional file 2: Figure S2. FISH mapping of DP-3 (b, g, and l; green) and DP-4 ( $c$, h, and $m$; red) by sequential FISH using $45 \mathrm{~S}$ rDNA (e, g, and $\mathrm{j}$; red), $5 \mathrm{~S}$ rDNA (e, g, and $\mathrm{j}$, green) and $A$. duranensis (a, green) and $A$. ipaënsis (a, red) total genomic DNA as probes in SLH $(a-e)$, A. duranensis $(f-j)$, and A. ipaënsis (k-o). (TIF $2188 \mathrm{~kb})$

Additional file 3: Figure S3. FISH mapping of DP-5 (b, g, and l; green) and DP-6 ( $c$, h, and $\mathrm{m}$; red) by sequential FISH using $45 \mathrm{~S}$ rDNA (e, $g$, and $\mathrm{j}$; red), $5 \mathrm{~S}$ rDNA (e, g, and j, green) and $A$. duranensis (a, green) and $A$. ipaënsis $(a$, red) total genomic DNA as probes in SLH $(a-e)$, A. duranensis $(\mathrm{f}-\mathrm{j})$, and A. ipaënsis $(\mathrm{k}-\mathrm{O})$. (TIF $2587 \mathrm{~kb}$ )

Additional file 4: Figure S4. FISH mapping of DP-8 (b, $g$, and l; green) and DP-7 ( $c, h$, and $m$; red) by sequential FISH using $45 \mathrm{~S}$ rDNA (e, g, and $\mathrm{j}$; red), $5 \mathrm{~S}$ rDNA (e, g, and $\mathrm{j}$, green) and $\mathrm{A}$. duranensis (a, green) and $A$. ipaënsis (a, red) total genomic DNA as probes in SLH (a-e), A. duranensis $(f-j)$, and A. ipaënsis $(\mathrm{k}-0)$. (TIF $2283 \mathrm{~kb})$
Additional file 5: Figure S5. Sequential FISH/GISH using Multiplex \#1 (left column, SSON), A. duranensis genomic DNA, A. ipaënsis genomic DNA (middle column, GISH), 45S rDNA, and 5S rDNA (right column, 45S/ 5S) as probes in eight peanut varieties. (TIF $2593 \mathrm{~kb}$ )

Additional file 6: Figure S6. Dual-color FISH using Multiplex \#1 in SLH $(a \sim d)$, A. duranensis $(e \sim h)$, and A. ipaënsis $(\mathrm{i} \sim \mathrm{l})$. (TIF $1882 \mathrm{~kb})$

Additional file 7: Figure S7. Sequential FISH using multiplex \#1 (left column, SSON), 45S rDNA, and $5 \mathrm{~S}$ rDNA (right column, 45S/5S) as probes in eight Arachis species. (TIF $3606 \mathrm{~kb}$ )

Additional file 8: Figure S8. Idiogram karyotypes of SLH and eight Arachis species. sm, submetacentric; st, subtelocentric; Bar, $3 \mu \mathrm{m}$. (TIF $666 \mathrm{~kb}$ )

Additional file 9: Figure S9. Karyotypes of two hybrid $F_{1}$-derived cultivated peanut and diploid wild species using repetitive Multiplex \#1 (SSON), $45 \mathrm{~S}$ and 5S rDNA (45S/5S) plasmid clones as probes. (TIF $1258 \mathrm{~kb})$

Additional file 10: Figure S10. FISH using oligonucleotide library 6A-1 in A. duranensis. (TIF $1066 \mathrm{~kb}$ )

Additional file 11: Figure S11. Sequential FISH using DP-5 (a and e, green), 45S rDNA ( $b$ and $f$, green), and 55 rDNA ( $c$ and g, green) in $A$. duranensis, and its merged figures ( $d$ and h). (TIF $1141 \mathrm{~kb}$ )

\section{Abbreviations}

BAC: Bacterial artificial chromosome; DAPI: 4', 6-diamidino-2-phenylindole; FAM: 6-carboxyfluorescein; FISH: Fluorescence in situ hybridization;

GISH: Genomic in situ hybridization; GSRs: Genomic sequence repeats; ITRs: Interstitial telomeric repeats. SSC: saline-sodium citrate; MCGSs: Multi-copy genetic sequences; SLH: Silihong; SSONs: Single-stranded oligonucleotides;" SSRs: Simple sequence repeats; TAMRA: 6-carboxytetramethylrhodamine

\section{Acknowledgments}

We thank Ms. Kassandra Semrau (MYcroarray, Ann Arbor, Michigan, USA) and Dr. Minfeng Xue (Institute for Plant Protection and Soil Science, Hubei Academy of Agricultural Sciences, Wuhan, China) for designing the chromosome-specific oligonucleotide library and genomic repeat sequence-based oligonucleotide probes for this study. We thank Prof. Robert McIntosh (University of Sydney, Australia) and Prof. Guohao He (Center for Plant Biotechnology, Tuskegee University, USA) for their kind reviews, suggestions, and editing, and Barbara Goodson, PhD, from Liwen Bianji, Edanz Group China (www.liwenbianji.cn/ac), for editing the English text of a draft of this manuscript.

Funding

This research was supported by the China Agriculture Research System (CARS-13); the Henan Provincial Agriculture Research System, China (S2012-05); and the Major Technology Research and Development Program of Henan Province, China (161100111000).

\section{Availability of data and materials}

All the data pertaining to the present study have been included in table and/or figure form in the manuscript and authors are pleased to share analyzed/raw data and plant materials upon reasonable request. Other datasets supporting the conclusions of this article are included within the article and its additional files.

\section{Authors' contributions}

$X Z, Z Q, B H, W D, F T$, and $L Z$ designed the experiments; PD, LL, YH, HL, LF, ZS, $L Q, C C, J X, X D, S H$, and ZZ performed the probes design, FISH and analyzed the data; $P D, Z Q, X Z$, and $Y H$ wrote the manuscript. All authors have read and approved the manuscript.

Ethics approval and consent to participate Not applicable.

\section{Consent for publication}

Not applicable.

\section{Competing interests}

The authors declare that they have no competing interests. 


\section{Publisher's Note}

Springer Nature remains neutral with regard to jurisdictional claims in published maps and institutional affiliations.

\begin{abstract}
Author details
'Industrial Crops Research Institute, Henan Academy of Agricultural Sciences/ Key Laboratory of Oil Crops in Huang-Huai-Hai Plains, Ministry of Agriculture/ Henan Provincial Key Laboratory for Oil Crops Improvement, Zhengzhou 450002, Henan, China. ${ }^{2}$ State Key Laboratory of Crop Genetics and Germplasm Enhancement, Nanjing Agricultural University, Nanjing 210095, China. ${ }^{3}$ Jiangsu Key Laboratory of Phylogenomics and Comparative Genomics, School of Life Sciences, Jiangsu Normal University, Xuzhou 221116, China. ${ }^{4}$ School of Life Sciences, Zhengzhou University, Zhengzhou 450001, Henan, China.
\end{abstract}

Received: 5 February 2018 Accepted: 3 October 2018

Published online: 17 October 2018

\section{References}

1. Stalker HT. Utilizing wild species for peanut improvement. Crop Sci. 2017;57: 1102-20.

2. Food and Agriculture Organization of the United Nations. http://www.fao. org/faostat/en/\#data/QC. Accessed 20 Jan 2018.

3. Robledo G, Seijo G. Species relationships among the wild B genome of Arachis species (section Arachis) based on FISH mapping of rDNA loci and heterochromatin detection, a new proposal for genome arrangement. Theor Appl Genet. 2010;121:1033-46.

4. Silvestri MC, Ortiz AM, Lavia GI. rDNA loci and heterochromatin positions support a distinct genome type for ' $x=9$ species' of section Arachis (Arachis, Leguminosae). Plant Syst Evol. 2015;301:555-62.

5. Raina SN, Mukai Y. Genomic in situ hybridization in Arachis (Fabaceae) identifies the diploid wild progenitors of cultivated (A. hypogaea) and related wild (A. monticola) peanut species. Plant Syst Evol. 1999;214:251-62.

6. Seijo JG, Lavia Gl, Fernández A, Krapovickas A, Ducasse D, Mmoscone EA. Physical mapping of the $5 \mathrm{~S}$ and 18S-25S rRNA genes by FISH as evidence that Archis duranensis and A. ipaënsis are the wild diploid progenitors of $A$. hypogaea (Leguminosae). Am J Bot. 2004;91:1294-303.

7. Robledo G, Lavia Gl, Seijo JG. Species relations among wild Arachis species with the Agenome as revealed by FISH mapping of rDNA loci and heterochromatin detection. Theor Appl Genet. 2009;118:1295-307.

8. Nielen S, Fonseca FC, Bertioli SL, Guimarães P, Seijo G, Town C, et al. FIDEL - a retrovirus-like retrotransposon and its distinct evolutionary histories in the A- and B-genome components of cultivated peanut. Chromosom Res. 2010;18:227-46.

9. Zhang L, Xu C, Yu W. Cloning and characterization of chromosomal markers from a Cot-1 library of peanut (Arachis hypogaea L.). Cytogenet Genome Res. 2012;137:1-41.

10. Du P, Li LN, Zhang ZX, Liu H, Qin L, Huang BY, et al. Chromosome painting of telomeric repeats reveals new evidence for genome evolution in peanut. J Integr Agr. 2016;15:2488-96.

11. Zhang LN, Yang XY, Tian L, Chen L, Yu WC. Identification of peanut (Arachis hypogaea) chromosomes using a fluorescence in situ hybridization system reveals multiple hybridization events during tetraploid peanut formation. New Phytol. 2016;211:1424-39.

12. Cuadrado A, Golczyk H, Jouve N. A novel, simple and rapid nondenaturing FISH (ND-FISH) technique for the detection of plant telomeres. Potential use and possible target structures detected. Chromosom Res. 2009;17:755-62.

13. Beliveau BJ, Joyce EF, Apostolopoulos N, Yilmaz F, Fonseka CY, McCole RB, et al. Versatile design and synthesis platform for visualizing genomes with oligopaint FISH probes. Proc Nail Acad Sci USA. 2012;109:21301-6.

14. Mikš-Krajnik M, Babuchowski A. 165 rRNA-targeted oligonucleotide probes for direct detection of Propionibacterium freudenreichii in presence of Lactococcus lactis with multicolour fluorescence in situ hybridization. Lett Appl Microbiol. 2014;59:320-7.

15. Han YH, Zhang T, Thammapichai P, Weng YQ, Jiang JM. Chromosomespecific painting in Cucumis species using bulked oligonucleotides. Genetics. 2015;200:771-9.

16. Du P, Zhuang LF, Wang YZ, Yuan L, Wang Q, Wang DR, et al. Development of oligonucleotides and multiplex probes for quick and accurate identification of wheat and Thinopyrum bessarabicum chromosomes. Genome. 2017;60:93-103.
17. Zhu MQ, Du P, Zhuang LF, Chu CG, Zhao H, Qi ZJ. A simple and efficient non-denaturing FISH method for maize chromosome differentiation using single-strand oligonucleotide probes. Genome. 2017;60:657-64.

18. Cuadrado A, Jouve N. Chromosomal detection of simple sequence repeats (SSRs) using nondenaturing FISH (ND-FISH). Chromosoma. 2010; 119:495-503.

19. Wang YZ. Development and characterization of small segment translocations of Thinopyrum bessarabicum and cytological mapping of interest genes. Nanjing: Master's thesis of Nanjing Agricultural University; 2013. In Chinese with English abstract

20. Tang ZX, Yang ZJ, Fu SL. Oligonucleotides replacing the roles of repetitive sequences pAs1, pSc1 19.2, pTa-535, pTa71, CCS1, and pAWRC.1 for FISH analysis. J Appl Genet. 2014;55:313-8.

21. Fu SL, Chen L, Wang YY, Li M, Yang Z. Oligonucleotide probes for NDFISH analysis to identify rye and wheat chromosomes. Sci Rep. 2015;5: 10552.

22. Beliveau BJ, Joyce EF, Apostolopoulosa N, Yilmaza F, Fonseka CY, McCole RB, et al. Oligopaints: highly efficient, bioinformatically designed probes for fluorescence in situ hybridization. Epigenet. Chromatin. 2013;6:5.

23. Danilova TV, Friebe B, Gill BS. Single-copy gene fluorescence in situ hybridization and genome analysis: Acc-2 loci mark evolutionary chromosomal rearrangements in wheat. Chromosoma. 2012;121:597-611.

24. Li KP, Wu YX, Zhao H, Wang Y, Lu XM, Wang JM, et al. Cytogenetic relationships among Citrullus species in comparison with some genera of the tribe Benincaseae (Cucurbitaceae) as inferred from rDNA distribution patterns. BMC Evol Biol. 2016;16:85.

25. Schimak MP, Kleiner M, Wetzel S, Liebeke M, Dubilier N, Fuchs BM. MiL-FISH: multilabeled oligonucleotides for fluorescence in situ hybridization improve visualization of bacterial cells. Appl Environ Micro. 2016;82:162-70.

26. Beliveau BJ, Boettiger AN, Avendaño MS, Jungmann R, McCole RB, Joyce EF, et al. Single-molecule super-resolution imaging of chromosomes and in situ haplotype visualization using oligopaint FISH probes. Nat Commun. 2015;6:7147.

27. Chen KH, Boettiger AN, Moffitt JR, Wang SY, Zhuang XW. Spatially resolved, highly multiplexed RNA profiling in single cells. Science. 2015;348(6233): aaa6090.

28. Moffitta JR, Hao JJ, Wang GP, Chen KH, Babcock HP, Zhuang XW. Highthroughput single-cell gene-expression profiling with multiplexed errorrobust fluorescence in situ hybridization. Proc Nail Acad Sci USA. 2016;113: 11046-51.

29. Bertioli DJ, Cannon SB, Froenicke L, Huang GD, Farmer AD, Cannon EK, et al. The genome sequences of Arachis duranensis and Arachis ipaensis, the diploid ancestors of cultivated peanut. Nat Genet. 2016; 48:438-46.

30. Zhao CZ, Qiu JJ, Agarwal G, Wang JS, Ren XZ, Xia H, et al. Genome-wide discovery of microsatellite markers from diploid progenitor species, Arachis duranensis and $A$. ipaënsis, and their application in cultivated peanut ( $A$. hypogaea). Front Plant Sci. 2017:8:1209-20.

31. Seijo G, Lavia Gl, Fernández A, Krapovickas A, Ducasse DA, Bertioli DJ, et al. Genomic relationships between the cultivated peanut (Arachis hypogaea, Leguminosae) and its close relatives revealed by double GISH. Am J Bot. 2007;94:1963-71.

32. Koppolu R, Upadhyaya HD, Dwivedi SL, Hoisington DA, Varshney RK. Genetic relationships among seven sections of genus Arachis studied by using SSR markers. BMC Plant Biol. 2010;10:15-27.

33. Mallikarjuna N. Production of hybrids between Arachis hypogaea and A. chiquitana (section Procumbentes). Peanut Sci. 2005;32:148-52.

34. He L, Liu J, Torres GA, Zhang HQ, Jiang JM, Xie CH. Interstitial telomeric repeats are enriched in the centromeres of chromosomes in Solanum species. Chromosom Res. 2013;21:5-13.

35. Marcais G, Kingsford C. Lock-free approach for efficient parallel counting of occurrences of k-mers. Bioinformatics. 2011;27:764-70.

36. PeanutBase. https://peanutbase.org/gbrowse_aradu1.0. Accessed $22 \mathrm{Nov}$ 2017

37. RepeatMasker. http://www.repeatmasker.org. Accessed 28 Nov 2017.

38. Kent WJ. BLAT - the BLAST-like alignment tool. Genome Res. 2002;12:656-64

39. Untergasser A, Cutcutache I, Koressaar T, Ye J, Faircloth BC, Remm M, et al. Primer3 - new capabilities and interfaces. Nucleic Acids Res. 2012; 40:e115.

40. Murgha YE, Rouillard JM, Gulari E. Methods for the preparation of large quantities of complex single-stranded oligonucleotide libraries. PLoS One. 2014;9:e94752. 
41. Wang $C T$, Huang $Y$, Yang $X D$, Jiang $Y$, Zhang JC, Chen DX, et al. Isolation of DNA from peanut: comparison between modified CTAB and high salt, low pH methods. J Peanut Sci. 2002;31:20-3 In Chinese with English abstract.

42. Chen CY, Barkley NL, Wang ML, Holbrook CC, Dang PM. Registration of purified accessions for the U.S. Peanut mini-Core germplasm collection. J PI Registr. 2014;8(1):77.

43. Krapovickas A, Gregory W. Taxonomia del genero Arachis (Leguminosae) Bonplandia. 1994;8:1-187.

Ready to submit your research? Choose BMC and benefit from:

- fast, convenient online submission

- thorough peer review by experienced researchers in your field

- rapid publication on acceptance

- support for research data, including large and complex data types

- gold Open Access which fosters wider collaboration and increased citations

- maximum visibility for your research: over $100 \mathrm{M}$ website views per year

At BMC, research is always in progress.

Learn more biomedcentral.com/submissions 